\title{
Pathways of Neutrophil Granulocyte Activation in Hereditary Angioedema with C1 Inhibitor Deficiency
}

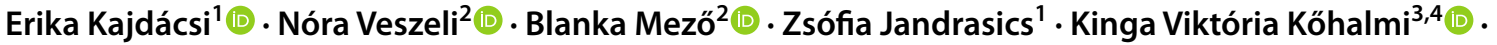 \\ Anne Lise Ferrara ${ }^{5}$ (D) László Cervenak ${ }^{1}$ (D) Lilian Varga $^{1,3}$ (D) Henriette Farkas ${ }^{1,3}$
}

Accepted: 26 January 2021 / Published online: 19 February 2021

(c) The Author(s) 2021

\begin{abstract}
Hereditary angioedema (HAE) with C1-inhibitor deficiency belongs to bradykinin-mediated angioedemas. It is characterized by recurrent subcutaneous and/or submucosal swelling episodes (HAE attacks) and erythema marginatum skin rash as a pre-attack (prodromal) phase. HAE attacks were shown to be accompanied by peripheral blood neutrophilia. We aimed to find molecular mechanisms that may explain the distinct role of neutrophil granulocytes in HAE. Plasma levels of blood cells and factors related to neutrophil activation (cytokines, chemokines, chemotactic factors, enzymes, and neutrophil extracellular trap) were measured in plasma samples obtained from patients during symptom-free periods $(n=77)$, during prodromal phase $(n=8)$ and attacks $(n=14)$, during a spontaneously resolved attack $(n=1)$, and in healthy controls $(n=79)$. Higher counts of white blood cells, lymphocytes, and neutrophil granulocytes were found in symptom-free patients compared with controls; these cell counts were elevated further during HAE attacks. The level of chemokine (C-C motif) ligand 5, monocyte chemoattractant protein-1, and myeloperoxidase were also higher in the symptom-free patients than in the controls. Levels of monocyte chemoattractant protein-1, leukotriene B4, neutrophil elastase, and myeloperoxidase were elevated during attacks. During erythema marginatum, white blood cells and monocyte count and levels of interleukin 8 were elevated compared with symptom-free period. Similar changes were detected during the attack follow-up. We conclude that the activation of NGs in symptom-free periods and a further increase observed during attacks suggests that NGs may be involved in the pathomechanism of HAE with C1-INH deficiency.
\end{abstract}

Keywords Hereditary angioedema $\cdot$ Erythema marginatum $\cdot$ Neutrophils $\cdot$ Cytokines $\cdot$ C1-inhibitor $\cdot$ Bradykinin

Henriette Farkas

farkas.henriette@med.semmelweis-univ.hu

Research Laboratory, Department of Internal Medicine and Hematology, Semmelweis University, 46 Szentkirályi str, 1088 Budapest, Hungary

2 MTA-SE Research Group of Immunology and Hematology, Hungarian Academy of Sciences and Semmelweis University, Budapest, Hungary

3 Hungarian Angioedema Center of Reference and Excellence, Department of Internal Medicine and Haematology, Semmelweis University, Budapest, Hungary

4 Department of Rheumatology, Hospital of Hospitaller Brothers of St. John of God, Budapest, Hungary

5 Center for Basic and Clinical Immunology Research (CISI), Department of Translational Medical Science, University of Naples "Federico II", Napoli, Italy

$\begin{array}{ll}\text { Abbreviations } & \\ \text { BK } & \text { Bradykinin } \\ \text { C1-INH } & \text { C1 esterase inhibitor } \\ \text { C1-INH-HAE } & \text { HAE with C1-INH deficiency } \\ \text { C1q, C3, C4 } & \text { Complement components } \\ \text { CCL5 } & \text { Chemokine (C-C motif) ligand 5 } \\ \text { COPD } & \text { Chronic obstructive airway disease } \\ \text { HAE } & \text { Hereditary angioedema } \\ \text { EM } & \text { Erythema marginatum } \\ \text { KKS } & \text { Kinin-kallikrein system } \\ \text { LTB4 } & \text { Leukotriene B4 } \\ \text { MCP-1 } & \text { Monocyte chemoattractant protein-1 } \\ \text { MPO } & \text { Myeloperoxidase } \\ \text { NE } & \text { Neutrophil elastase } \\ \text { NET } & \text { Neutrophil extracellular trap } \\ \text { NGC } & \text { Neutrophil granulocyte count } \\ \text { NGs } & \text { Neutrophil granulocytes } \\ \text { PKa } & \text { Plasma kallikrein } \\ \text { PRTN3 } & \text { Proteinase 3 }\end{array}$


TNF $\alpha \quad$ Tissue necrosis factor alpha

VAS Visual analog scale

WBC White blood cell

\section{Introduction}

Hereditary angioedema (HAE) due to C1-inhibitor (C1-INH) deficiency (C1-INH-HAE) is a rare, potentially lifethreatening disorder characterized by recurrent swelling episodes in subcutaneous and/or submucosal tissues, which are often preceded by prodromal symptoms [1, 2]. Forty-two to $58 \%$ of C1-INH-HAE patients have erythema marginatum (EM) as the only objective prodromal symptom of the HAE attacks $[3,4]$. Mutations in the SERPING1 gene results in decreased C1-INH function (type 1 low C1-INH, type 2 normal or increased C1-INH protein level) [5]. C1-INH is involved in the regulation of the complement, kallikrein-kinin, coagulation, and fibrinolytic plasma enzyme systems. The impairment of C1-INH function together with certain trigger factors can activate these cascade systems. The activation of the kallikrein-kinin system (KKS) results in cleavage of bradykinin (BK) from high molecular weight kininogen by plasma kallikrein (PKa). The release of BK increases vascular permeability, which causes angioedema by interacting with $\mathrm{BK}$ receptor $\mathrm{B} 2$ on the endothelium [6]. A number of articles have been published to explore the pathophysiology of C1-INH-HAE, focusing on the role of plasma enzyme systems and endothelial cells [7-9]. Increased white blood cell (WBC) count was observed during HAE attacks without apparent inflammatory signs. It was hypothesized that this is a consequence of hemoconcentration due to increased plasma extravasation of fluid into the extracellular space [10-12]. Zotter et al. observed that all hematologic values are increased during attacks and the elevation of WBC count is significantly greater ( 1.5-fold) than expected based on hemoconcentration alone, and it involves specifically the peripheral blood neutrophil granulocytes (NGs) [13]. A further study showed that neutrophil granulocyte count (NGC) is higher in C1-INHHAE patients during symptom-free periods than in healthy controls, and this difference is further emphasized during HAE attacks. Moreover, NGs were shown to be activated during edematous episodes [14]. It was proposed that release of NGs into the circulation contributes to the progression of HAE attacks, which may be precipitated by these cells themselves. NGs may contribute to the dysregulation of KKS, since this system can also be activated on the surface of neutrophils [15]. NGs are capable of producing cytokines and certain enzymes, and cytokines and chemokines produced by other cell types affect their functions. NGs produce neutrophil elastase (NE), myeloperoxidase (MPO), and proteinase 3 (PRTN3) in their azurophilic granules [16]. During activation of NGs, NE released from these cells may modify the C1-INH molecule and thereby further attenuate its already impaired function $[17,18]$. MPO is a local mediator of tissue damage and the resulting inflammation in various inflammatory diseases [19, 20]. The primary biological function of PRTN3 depends on its proteolytic activity, which is the degradation of extracellular proteins at inflammation sites [21]. The activation of NGs may lead to the formation of neutrophil extracellular traps (NETs) during which NGs release their DNA content and citrullinated histones decorated with the proteins from azurophilic granules (e.g., NE and MPO), specific granules, tertiary granules, and the cytoplasm to bind pathogens and also provide a negatively charged surface for the activation of the KKS and the complement systems [22-26].

Moreover, activated endothelial cells and platelets may assist to the formation of NETs and the activation of NGs by producing pro-inflammatory cytokines. TNF alpha (TNF $\alpha$ ), IL-8, and leukotriene B4 (LTB4) produced by NGs have autocrine effect on NG function itself $[27,28]$. TNF $\alpha$ is a cytokine involved in acute phase reactions and systemic inflammation. It is a potent chemoattractant for NGs, inducing their migration to the tissues by promoting the expression of adhesion molecules on endothelial cells [29]. Furthermore, TNF $\alpha$ can induce IL-8 production from a variety of cells (e.g., endothelial cells, macrophages, neutrophils, and epithelial cells) [28]. IL-8 is a neutrophil chemotactic factor which induces chemotaxis, primarily of NGs to the site of infection $[28,30]$. LTB4 also acts on NGs to elicit their chemotaxis and integrin-mediated adhesion to the vascular endothelium, thereby allowing them to bind to and cross into the tissue, and induces the formation of reactive oxygen species and the release of lysosomal enzymes [31].

There is limited data on levels of peripheral blood NGs and their function in C1-INH-HAE patients. Moreover, there is very limited data on the activation of these cells during HAE attack, or their activation during EM. The latter has not been investigated until now.

The objectives of our study were (1) to confirm our previous results, on higher NGC in patients compared with healthy controls, and elevated NGC in patients during attacks, as compared with symptom-free periods; (2) to find a molecular pattern among neutrophil chemoattractants and activation markers, which may explain the distinct behavior of NGs in HAE patients; and (3) to map the neutrophil function during EM and the kinetic changes during an HAE attack, followed from the beginning until its spontaneous termination.

\section{Study Population}

\section{Patients}

The patients were selected from the total C1-INH-HAE patient population $(n=197)$ receiving regular care at the Hungarian Angioedema Center of Reference and Excellence. 
The diagnosis of C1-INH-HAE was established by family history, evaluation of the clinical manifestations, and laboratory parameters (antigenic and functional levels of C1-INH, $\mathrm{C} 1 \mathrm{q}$, and $\mathrm{C} 4)$. We included 77 consecutive patients who presented at the center for follow-up visits without HAE symptoms during 2014 and 2018, 14 patients with HAE attack, and 8 patients having prodromal (EM) without attack. A subcutaneous HAE attack was followed from the beginning until its spontaneous termination in one 56-year-old female patient with C1-INH-HAE type I [32]. None of the patients had any clinical manifestation suggestive of an acute infection at the time of blood sampling.

\section{Healthy Controls}

Healthy control group (gender and aged-matched) consisted of 79 healthy adults and one distinct gender- and agedmatched control for the HAE patient, who were studied in the kinetic follow-up part of the study. The healthy controls did not have any known medical diagnoses and did not receive medications at the time of blood sampling. C1-INH deficiency was excluded by complement testing (see above). Detailed demographic data of the patients and controls are summarized in Table 1 and Fig. 1 depicts the patient and control cohorts.

\section{Methods}

\section{Blood Sampling}

Peripheral blood samples were obtained by venipuncture from patients with C1-INH-HAE during symptom-free periods, HAE attacks (before acute treatment), and EM, as well as from healthy subjects. Samples from the symptomfree periods were collected from patients who had been symptom-free for at least $72 \mathrm{~h}$ before blood sampling and had not received acute treatment for their HAE attack. The mean latency between the detection of the HAE symptoms and blood sampling was $7.8 \mathrm{~h}$ (SD: 7.6). The mean interval between symptom-free and during-an-attack sampling in the same patients was 9.5 months (SD: 7.7).
During the HAE attack kinetic follow-up part of the study, EDTA-plasma samples were collected 12 consecutive times, over $96 \mathrm{~h}$ of observation during an HAE attack in a single case. The patient rated the severity of her symptoms on a visual analog scale (VAS) More clinical details of this HAE attack have been described previously by Veszeli et al. [32].

EDTA plasma and serum samples were stored at $-80^{\circ} \mathrm{C}$ until processing.

\section{Measurement of Blood Cell Count and Factors Related to Neutrophil Activation}

WBC, NGs, lymphocyte, thrombocyte, monocyte, eosinophil, and basophil granulocyte counts were determined in the samples by using Advia120 Hematology System automate (Siemens, Erlangen, Germany). To determine the levels of the enzymes NE, MPO, PRTN3, as well as cytokines [TNF- $\alpha$, IL-8, MCP-1, CCL5], and LTB4 levels, we used commercially available ELISA KITs. As negative control, we measured monocyte chemoattractant protein-1 (MCP-1) and chemokine (C-C motif) ligand 5 (CCL5) levels. MCP-1 is a chemoattractant and activating factor for monocytes, basophils, T cells, NK cells, and immature dendritic cells. $\mathrm{MCP}-1$, in general, is considered as a chemotactic factor for monocytes and basophils but not neutrophils or lymphocytes are affected [34]. CCL-5 is a chemotactic stimulus for T cells, eosinophils, and basophils and plays an important role in attracting other types of leukocytes to inflammatory sites. The following ELISA KITs were used: IL-8 (KHC0083), NE (BMS269), and TNF $\alpha$ (KHC3014) from Thermo Fisher Scientific; LTB4 (KGE006B), CCL5 (DY278-05), and PRTN3 (DY6134-05) from R\&D Systems; MPO (K 6631B) from Immundiagnostik; and MCP-1 (ab179886) from Abcam, according to the manufacturer's instructions.

To measure NETs, we developed an in-house ELISA method. Briefly, anti-MPO antibody (PA5-16,672, Thermo Fisher Scientific) was used for capturing; for detection, we used anti-dsDNA antibody (ab27156, Abcam). The 96-well microtiter plates (Nunc ${ }^{\circledR}$ Maxisorp ${ }^{\circledR}$ ) were coated with capture antibody 1:2000 overnight on $4{ }^{\circ} \mathrm{C}$. After the blocking step ( $1 \mathrm{~h}$ room temperature, with $2 \%$ BSA-PBS), the standard and the samples (fourfold dilution) were incubated on

Table 1 Summary of the basic data of C1-INH-HAE patients and healthy controls

\begin{tabular}{|c|c|c|c|c|}
\hline & \multirow{2}{*}{$\begin{array}{l}\text { Healthy controls } \\
(n=79)\end{array}$} & \multicolumn{3}{|l|}{ C1-INH-HAE patients } \\
\hline & & Symptom-free $(n=77)$ & HAE attack $(n=14)$ & EM prodrome $(n=8)$ \\
\hline Male/female $(n)$ & $34 / 45$ & $37 / 40$ & $6 / 8$ & $1 / 7$ \\
\hline $\begin{array}{l}\text { Age (years): median; } 25-75 \% \\
\text { percentile }\end{array}$ & $38 ; 32-46$ & $41 ; 28.5-52$ & $37.5 ; 27.3-49.3$ & $40.5 ; 25.8-48.5$ \\
\hline C1-INH-HAE type I/II $(n)$ & - & $70 / 7$ & $11 / 3$ & $8 / 0$ \\
\hline
\end{tabular}




\section{a}

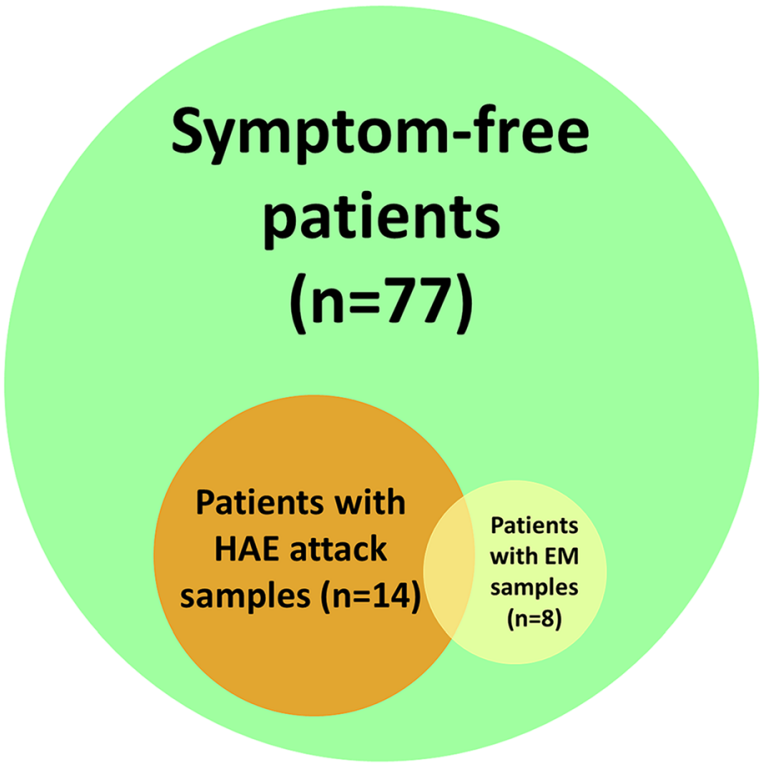

b

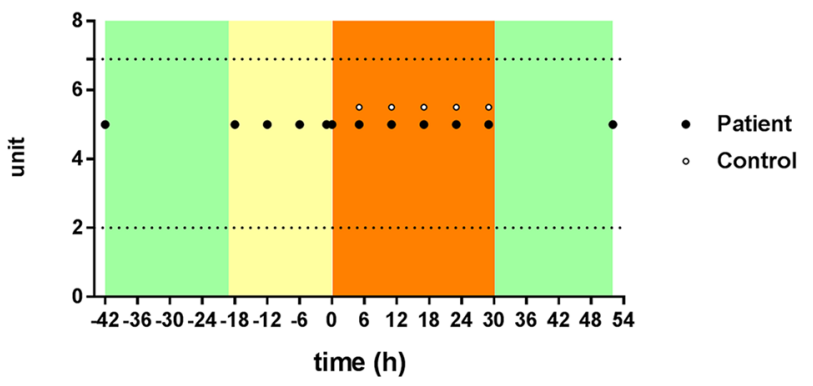

Fig. 1 Disposition of subjects in the study. a We made three types of comparisons: controls $(n=79)$ with symptom-free patients $(n=77)$, symptom-free samples with the patients' own attack samples $(n=14)$, and symptom-free samples with the patients' own EM (prodromal) samples $(n=8)$. b A 56-year-old female patient with type I C1-INH-

the plate overnight on $4{ }^{\circ} \mathrm{C}$. The next day, after washing the plate 3 times with PBS with $0.05 \%$ Tween 20 , the detection antibody was incubated for $1 \mathrm{~h}$ on room temperature (1:4000). After washing 3 times, goat anti-mouse HRPconjugated secondary antibody was used $(1 \mathrm{~h}$, room temperature, 1:2000, Southern Biotechnology). After 4 times washing, TMB substrate (Life Technologies) was used. The reaction was terminated with $1 \mathrm{M} \mathrm{H}_{2} \mathrm{SO}_{4}$, and $\mathrm{OD}$ was determined with a microplate reader (Infinite ${ }^{\circ}$ M1000 PRO, Tecan Group Ltd) at 460/620 nm. The samples were calculated from the standard curve. The standard curve was generated from freshly isolated NGs from a healthy volunteer $\left(10 \times 10^{6}\right.$ cells $/ 2 \mathrm{ml}$ HBSS $+20 \mathrm{mM}$ HEPES buffer $)$ activated with $100 \mathrm{nM}$ PMA on $37{ }^{\circ} \mathrm{C}$ during a 3-h period. After incubation, the cells were centrifuged and the supernatant was collected and used as NET standard in 1/500 dilution, followed by 2 times linear dilution.
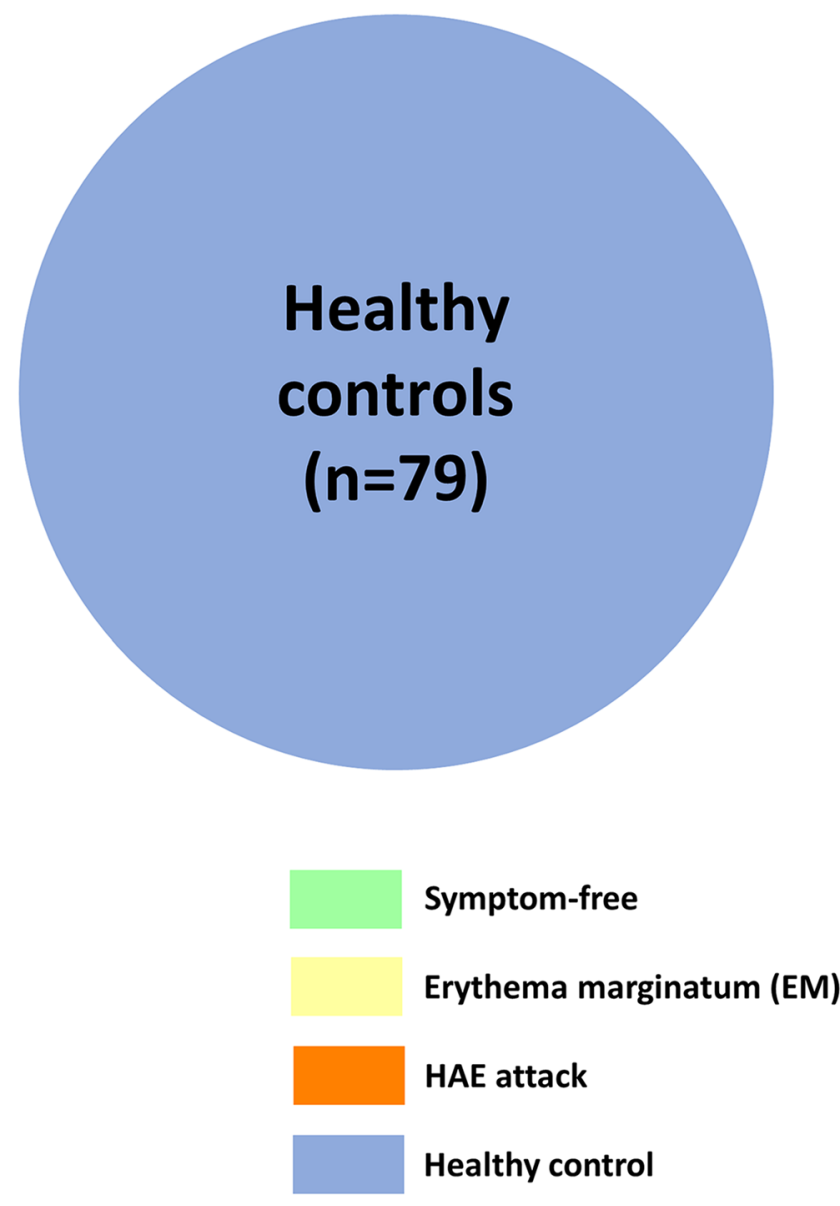

HAE was followed from her symptom-free phase (green), during EM (prodrome) (yellow) and angioedema attack (orange) until remission. Twelve EDTA-plasma samples were collected from the patient (solid circles) and 5 from the control person (open circles). Circles indicate sampling times. Normal range is represented by dotted lines

All laboratory tests were performed on freshly thawed plasma samples. All samples were measured in single assessments (with the re-measuring of some samples).

The study protocol was approved by the institutional review board of Semmelweis University of Budapest, and informed consent was obtained from all the participants in accordance with the Declaration of Helsinki.

\section{Statistical Analysis}

The results of ELISA measurements were analyzed and interpreted using GraphPad Prism v7.00 (GraphPad Software Inc.). For statistical analysis, Mann-Whitney test, unpaired $t$ test, paired $t$ test, and Pearson's $r$ correlation test were used. 


\section{Results}

The main purpose of this study was to investigate the levels of peripheral blood NGs and the parameters related to their activation in C1-INH-HAE.

\section{Peripheral Blood Cell Counts and Plasma Levels of Factors Related to Neutrophil Activation in C1-INH-HAE Patients and Healthy Controls}

Total WBC and different blood cell type counts were compared in peripheral blood samples obtained from 79 healthy subjects and 77 C1-INH-HAE patients. Total number of WBCs, lymphocytes, NGs, and basophil granulocytes was significantly higher in symptom-free C1-INH-HAE patients than in healthy controls (Table 2).

The neutrophil-related factors: CCL5, MCP-1, and MPO were significantly higher in patients during symptom-free periods than in healthy controls (Table 3).

\section{Peripheral Blood Cell Counts and Factors Related to Neutrophil Activation in C1-INH-HAE Patients During EM and HAE Attacks}

Out of 77 C1-INH-HAE patients, plasma samples were collected from 8 patients during EM and from 14 patients during HAE attacks (Fig. 1). The body locations of the HAE attacks were subcutaneous in 8 patients, abdominal in 3 patients, and combined subcutaneous and abdominal in 3 patients. Peripheral blood cell types and neutrophil-related parameters in these 2 subgroups were compared:

WBC and monocyte cell counts were significantly elevated during $\mathrm{EM}$ (WBC: $8.4710^{9} / \mathrm{L} \pm 1.29$, monocytes: 0.5 $10^{9} / \mathrm{L} \pm 0.16$ ) compared with symptom-free periods (WBC: $7.2110^{9} / \mathrm{L} \pm 1.22$, monocytes: $\left.0.410^{9} / \mathrm{L} \pm 0.13\right)(p=0.0272$
Table 3 Factors related to neutrophil activation in healthy controls and C1-INH-HAE patients during symptom-free periods

\begin{tabular}{llll}
\hline & $\begin{array}{l}\text { Healthy controls } \\
(n=79)\end{array}$ & $\begin{array}{l}\text { C1-INH-HAE } \\
\text { symptom-free } \\
\text { period }(n=77)\end{array}$ & $P$ value \\
\hline PRTN3 (ng/ml) & $19.7(14.6-28.69)$ & $23.2(16.2-27.8)$ & $\mathrm{ns}$ \\
CCL5 $(\mathrm{ng} / \mathrm{ml})$ & $12.4(5.1-24.7)$ & $19.2(10.2-28.7)$ & 0.0076 \\
TNF a $(\mathrm{pg} / \mathrm{ml})$ & $12.4(8.1-14.8)$ & $9.9(8.1-14.1)$ & $\mathrm{ns}$ \\
$\mathrm{IL}-8(\mathrm{pg} / \mathrm{ml})$ & $0.94(0.41-1.66)$ & $1.01(0.6-1.43)$ & $\mathrm{ns}$ \\
MCP-1 (pg/ml) & $54(44.6-73.7)$ & $64.5(52-83.2)$ & 0.0288 \\
NE $(\mathrm{ng} / \mathrm{ml})$ & $31.6(24.5-40.9)$ & $29.9(22.6-37.6)$ & $\mathrm{ns}$ \\
NET $(\mathrm{activated}$ & $159.3(132.4-191.4)$ & $158.7(139.2-190.9)$ & $\mathrm{ns}$ \\
cells/100 $\mu \mathrm{l})$ & & & \\
MPO $(\mathrm{ng} / \mathrm{ml})$ & $72.6(54.6-90.1)$ & $77.4(64.4-102.1)$ & 0.0478 \\
LTB4 $(\mathrm{ng} / \mathrm{ml})$ & $61.5(45.1-110.3)$ & $63(41-158.1)$ & $\mathrm{ns}$ \\
\hline
\end{tabular}

Ns: non-significant

Values are medians (25-75\% percentile). $P$ values were calculated with Mann-Whitney test

and $p=0.0169$, respectively, paired $t$ test). Among the neutrophil-related parameters, only the level of IL-8 was elevated during $\mathrm{EM}(1.66 \mathrm{pg} / \mathrm{ml} \pm 0.93)$ compared with symptom-free period $(0.88 \mathrm{pg} / \mathrm{ml} \pm 0.30),(p=0.0481$, paired $t$ test). The results are presented as means and \pm SD.

WBC, lymphocytes, and NGs count were significantly elevated during HAE attacks (Fig. 2a) compared with symptom-free periods. Additionally, neutrophil-related factors: MCP-1, NE, MPO, and LTB4 levels were significantly elevated during HAE attacks compared with symptom-free periods (Fig. 2b). Furthermore, we found correlations between the NGC and levels of PRTN3 $(r=0.7066$, $p=0.0047)$, CCL5 $(r=0.5968, p=0.0243)$, MCP-1 $(r=0.749, p=0.0021), \mathrm{NE}(r=0.9353, p<0.0001)$, and NET $(r=0.7626, p=0.0022)$.

Table 2 Peripheral blood cell type count in samples obtained from healthy subjects and C1-INH-HAE patients during symptom-free periods

\begin{tabular}{llllc}
\hline & Healthy controls $(n=79)$ & $\begin{array}{l}\text { C1-INH-HAE symptom-free } \\
\text { period }(n=77)\end{array}$ & $P$ value & Normal range \\
\hline White blood cell $\left(10^{9} / \mathrm{L}\right)$ & $6.38(5.1-7.4)$ & $7.25(6.3-8.5)$ & 0.0013 & $4.00-10.00$ \\
Lymphocyte $\left(10^{9} / \mathrm{L}\right)$ & $1.92(1.5-2.3)$ & $2.14(1.9-2.6)$ & 0.0010 & $1.00-4.00$ \\
Thrombocyte $\left(10^{9} / \mathrm{L}\right)$ & $244.5(215.3-286.8)$ & $262.5(224-306)$ & $\mathrm{ns}^{\mathrm{a}}$ & $\mathrm{ns}$ \\
Monocyte $\left(10^{9} / \mathrm{L}\right)$ & $0.35(0.28-0.42)$ & $0.35(0.3-0.43)$ & 0.0029 & $0.15-0.90$ \\
Neutrophil granulocyte $\left(10^{9} / \mathrm{L}\right)$ & $3.78(2.82-4.82)$ & $4.34(3.57-5.5)$ & $\mathrm{ns}$ & $0.00-6.90$ \\
Eosinophil granulocyte $\left(10^{9} / \mathrm{L}\right)$ & $0.125(0.04-0.18)$ & $0.13(0.08-0.16)$ & 0.0123 & $0.01-0.20$ \\
Basophil granulocyte $\left(10^{9} / \mathrm{L}\right)$ & $0.03(0.01-0.04)$ & $0.04(0.02-0.05)$ & & 0.0123 \\
\hline
\end{tabular}

Ns non-significant

Values are medians (25-75\% percentile). $P$ values were calculated with Mann-Whitney test in most cases

${ }^{\mathrm{a}}$ Unpaired $t$ test 

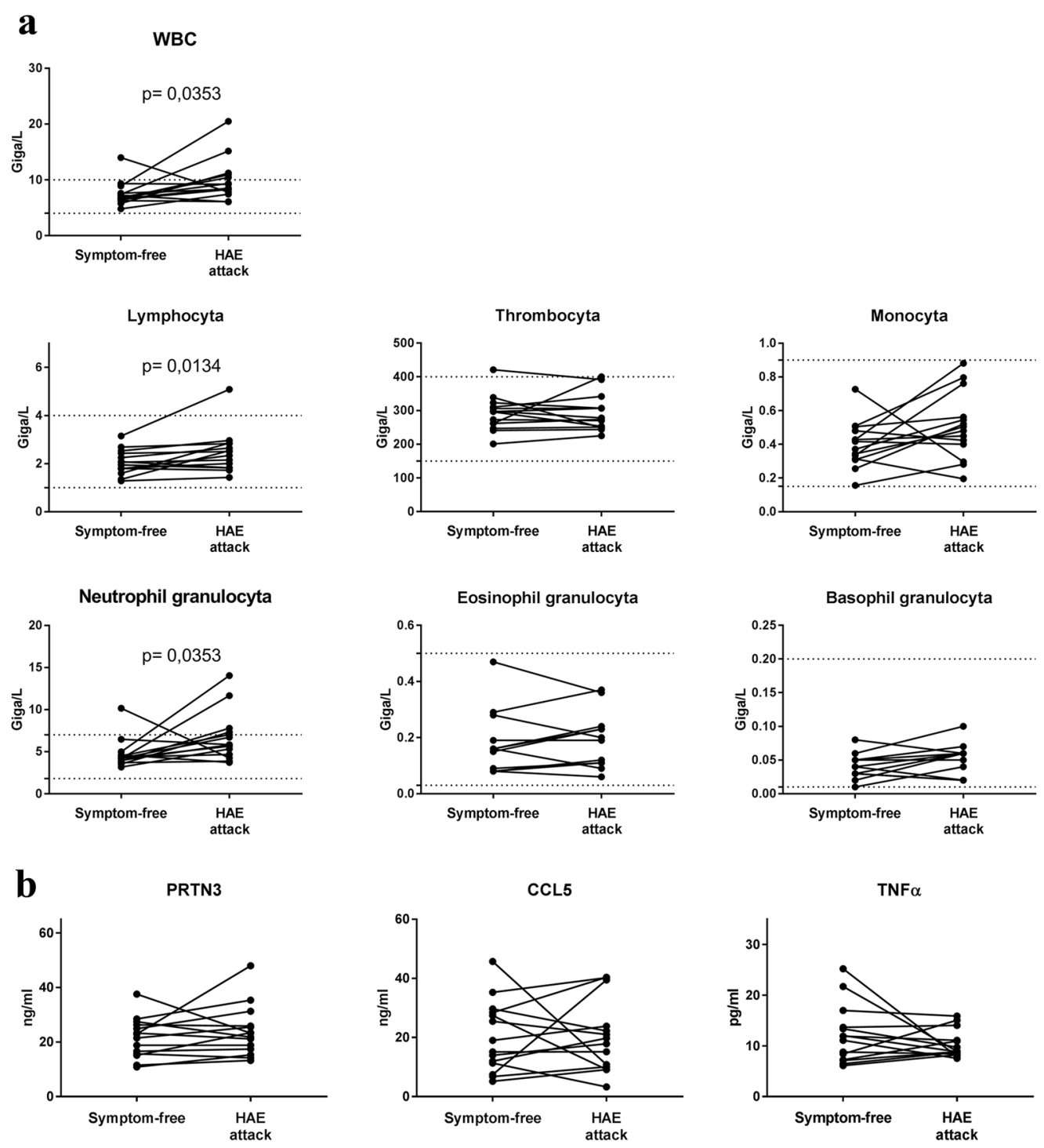

CCL5
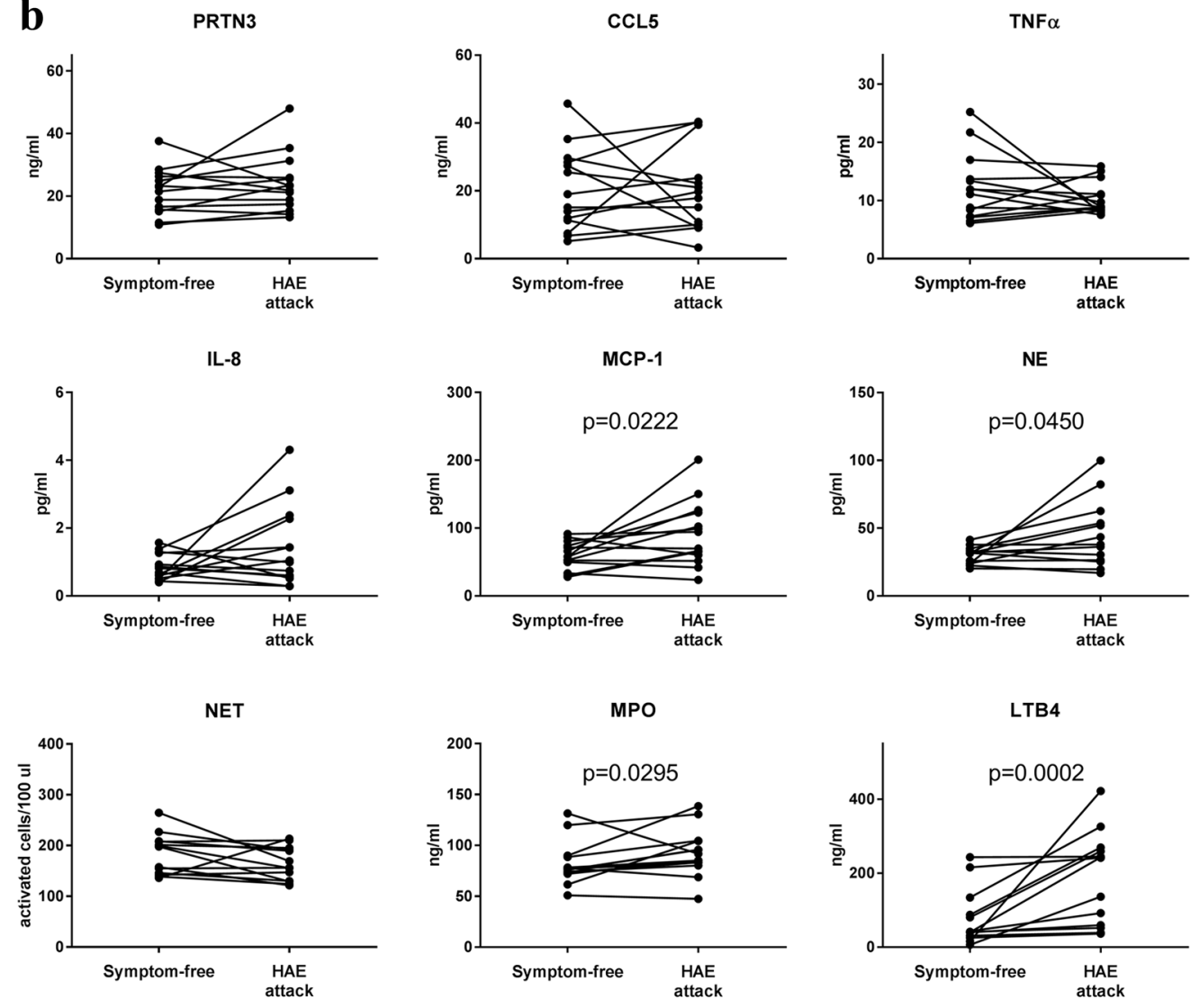
4Fig. 2 Blood cell count and factors related to neutrophil activation in HAE attacks compared with symptom-free periods. a WBC, thrombocyte, lymphocyte, and neutrophil-, eosinophil-, and basophil granulocyte count were measured in peripheral blood samples obtained from 14 patients in symptom-free periods and during HAE attacks. The dotted lines indicate normal range. Wilcoxon test (WBC, lymphocyte, neutrophil-, and eosinophil granulocyte) and paired $t$ test (thrombocyte, monocyte, basophil granulocyte) calculations were used. b Levels of neutrophil related factors were measured from plasma samples of 14 patients in symptom-free periods and during HAE attacks. Wilcoxon test (TNF $\alpha$, IL-8, MPO, LTB4) and paired $t$ test (PRTN3, CCL5, MCP-1, NE, NET) were used

\section{Kinetics of Peripheral Blood Cell Counts and Factors Related to Neutrophil Activation During HAE Attack}

WBC and NG cell counts were elevated in one patient observed all through an HAE attack concurrent with severity of symptoms, as indicated by VAS score, $6-12 \mathrm{~h}$ after the beginning of the attack. During the follow-up, we observed two peaks of monocyte cell count, one during prodromal phase (characterized by EM) and the other during the attack phase (Fig. 3a). Neutrophil-related factors: IL-8, NET, and PRTN3 showed also a distinct peak during the prodromal (EM) phase and 18- and $24 \mathrm{~h}$ after the beginning of the attack, respectively (Fig. 3b).

\section{Discussion}

Besides being the frontline cellular arm of the innate immunity against bacteria and fungi, NGs are able to actively regulate certain plasma enzyme cascade systems by producing and activating their components, as well as by forming NET as a negatively charged surface [33]. This is also true contrariwise: activation of plasma enzyme cascades significantly regulates the function of NGs at multiple levels. Therefore, we aimed to map the role of NGs in C1-INH-HAE, where the disturbance of plasma enzyme cascade systems (i.e., KKS, complement, fibrinolysis) is known. For better understanding, we summarized the between-group differences in Table 4.

\section{Cellular Alterations}

In C1-INH-HAE patients, an elevated WBC count was found during the prodromal (EM) and HAE attack phases; the composition of this cell population was different in the two phases. EM was characterized predominantly by elevated monocyte count, whereas HAE attacks NGC were elevated, as compared with symptom-free periods. Stress and physical exercise are known trigger factors of increased total WBC and NG counts as well as HAE attacks [34-36]. We cannot exclude that the increased WBC and NGC were caused by these trigger factors during the HAE attacks. However, elevated NGC and WBC were also found in symptom-free patients compared with healthy controls, which may suggest a stress or physical exercise independent elevation of cell counts at least in symptom-free period.

Elevated lymphocyte count was observed, in symptomfree patients compared with controls and also during HAE attack compared with symptom-free periods. Furthermore, monocyte count was also elevated during EM compared with symptom-free periods (also in the kinetic follow-up part). However, since our present study focused on the role of NGs in C1-INH-HAE, these results can be the basis for a future research.

\section{Chemotactic Factors}

Only two of the chemotactic factors that have effects on neutrophil cells are found elevated: IL-8 and LTB4. IL-8 was elevated in C1-INH-HAE during prodromal (EM) phase compared with symptom-free periods, and we could see the same elevation in the case of IL-8 in the kinetic followup part's prodromal phase that we have seen in the case of monocyte count. Monocytes are capable of producing IL-8, so we may assume that the elevation of these two factors be related. Monocytes are capable of producing IL-8 upon physical contact with endothelial cells, and this response is further augmented by pretreatment of endothelial cells with interferon gamma $[37,38]$. Therefore, it is possible that elevated monocyte count and/or the interaction between monocytes and endothelial cells are accounted for the elevated IL-8 level during prodromal EM. IL-8 also plays a key role in NG recruitment and degranulation [39]. Therefore, this may suggest that these alterations are initiated at an early pre-attack (prodromal) stage and continue into the clinical attack.

Plasma LTB4 was also elevated during attacks compared with symptom-free periods. LTB4 enhances the adhesion of the leukocytes to the endothelium-reinforced by activated endothelial cells [40]—and may potentiate NGs to attach and migrate into the tissues. This process seems to contradict the increased NGC in the blood. We do not know how many NGs bind to endothelial cells or how many migrate and become sequestered in the tissues, but the elevated plasma levels of LTB4 raises the possibility that we underestimate the levels of NGs and its activation during HAE attacks.

\section{Activated NG Products}

From the factors produced by activated NGs, all four were found elevated. MPO level was higher in symptom-free patients compared with healthy controls and was also elevated further during HAE attacks. Thus, in symptom-free periods, in spite of the higher NG numbers, their activation is only partially expressed. During HAE attacks, further elevation of the MPO level, parallel with elevation of NGC, 
a

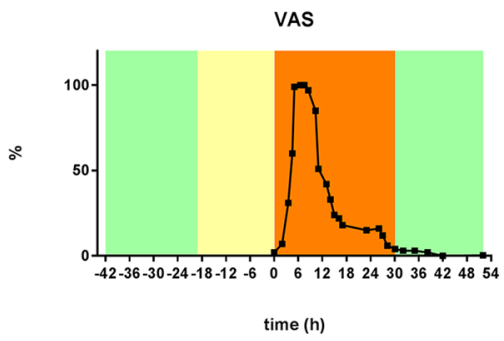

Lymphocyte
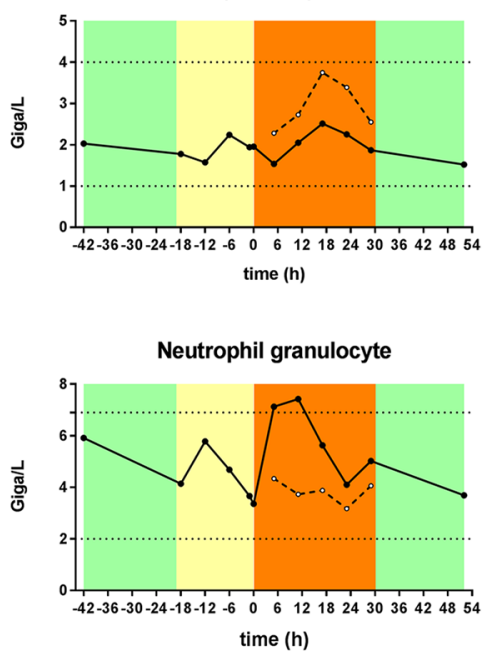

b

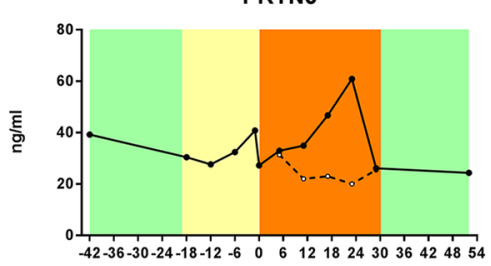

time (h)

IL-8

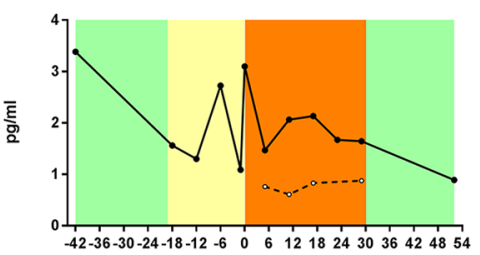

time (h)

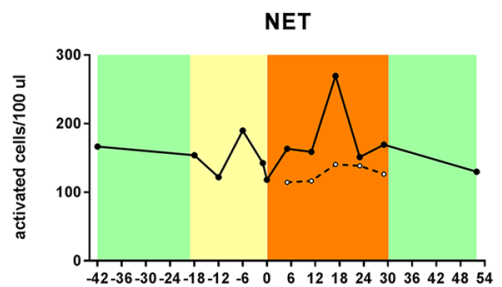

time (h)
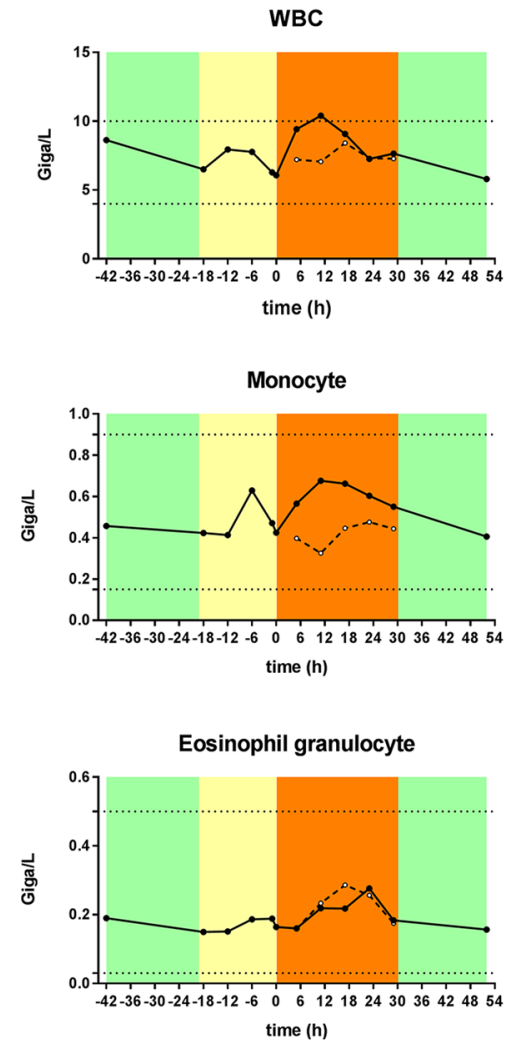

CCL5

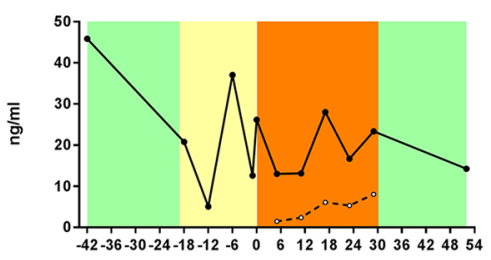

time (h)

MCP-1

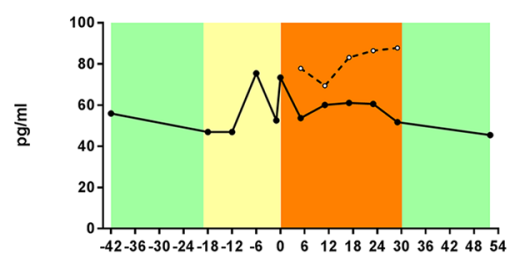

time (h)

LTB4

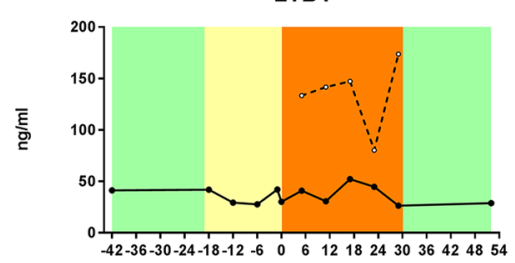

time (h)
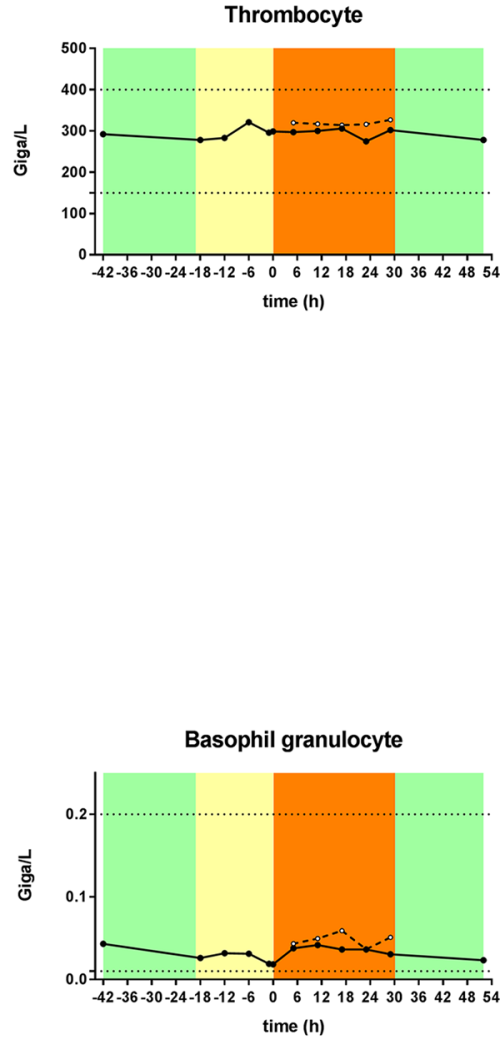

TNF $\alpha$

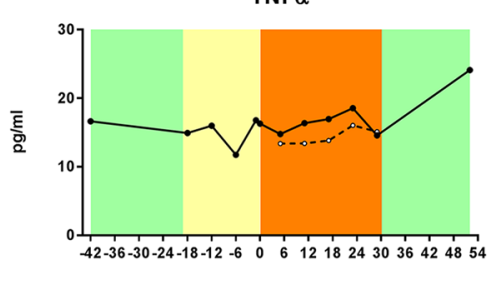

time (h)

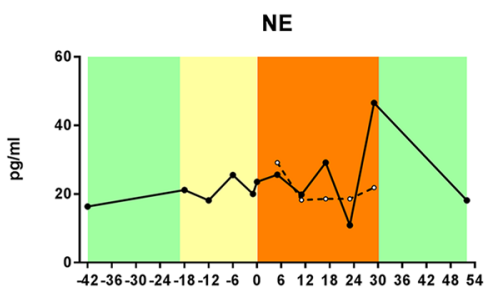

time (h)

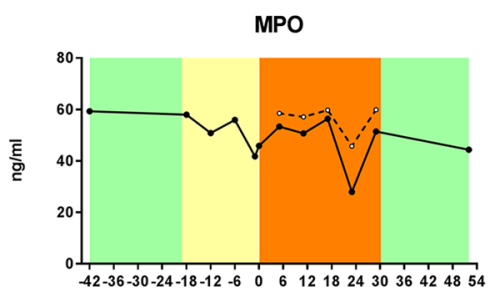

time (h) 
4Fig. 3 Kinetics of blood cell counts and factors related to neutrophil activation during an HAE attack (single patient). a Kinetics of $W B C$, thrombocyte, lymphocyte, and neutrophil-, eosinophil-, and basophil-granulocyte count during an HAE attack of a C1-INH-HAE patient (solid line with closed dots), and an age- and gender-matched healthy control (dashed line with open dots). Severity levels of angioedema symptoms were assessed by the patient in on a VAS scale $(0-100 \mathrm{~mm})$. Symptom-free periods are marked with green, prodromal phases with yellow, and attack phases with orange. The dotted lines indicate normal ranges. b Kinetics of neutrophil-related factors of the same patient and control

occurred, and the level of NE was also elevated compared with symptom-free periods. In the kinetic follow-up part of the study, levels of PRTN3 and NET were elevated only during HAE attack period. All these results and the strong correlation found between NGC and PRTN3, CCL5, MCP$1, \mathrm{NE}$, and NET further support that NGs are activated during HAE attacks. Activation of the KKS can occur on the surface of NGs and NET [15, 25, 26]. On the other hand, NGs can be activated by kinins formed during inflammation [41]. In a previous study [42], elevated kallikrein/ C1-INH complexes were found in C1-INH-HAE patients during symptom-free periods compared with healthy controls and also at the onset of HAE attacks (kinetic followup part). This suggests a correlation between activation of NGs and activation of the KKS. However, the question emerges, whether the NGs or its NET product induce KKS activation during HAE attacks, or conversely, the activation of the KKS attracts and activates NGs.

\section{Other Factors}

Both CCL5 and MCP-1 levels were higher in symptom-free patients compared with healthy controls, and MCP-1 levels was further elevated during HAE attacks. These results suggest that CCL5 and MCP-1 may also act on NGs and could explain the higher/elevated NG levels in C1-INH-HAE patients. Moreover, Balamayooran et al. found that MCP-1 has direct and indirect effects on NG migration in mice [43], and a similar result was found in the relation of CCL5 by Stefano et al. in chronic obstructive airway disease (COPD) patients [44].

\section{The Contribution of the Study}

There is relatively limited data about NGs and factors related to neutrophil activation in C1-INH-HAE patients. We propose that the higher level of NGs in the symptom-free stage of the patients may contribute to the constant activation of the KKS [42]. In contrast to previous studies [14, 45], we could not find any significant difference in IL-8 levels-one of the best-known chemotactic factors for NGs-between patients and healthy controls. Interestingly, we found that CCL5 and MCP-1 may have an important role in NG recruitment in HAE. Furthermore, we succeeded in adding a novel aspect: recruitment of the NGs during the early, prodromal (EM) phase [3, 46, 47]—concurrently with elevated monocyte count and elevated IL-8 level, which presumably originate from monocytes. Our observation supports the hypothesis that EM is an early phase of an HAE attack. This might provide an objective basis for new, individualized therapeutic strategies, such as administering acute treatment for HAE as early as the prodrome $[3,46]$.

During HAE attack, NGC increases, and NG activation also takes place, which can further enhance the KKS activation to maintain/contribute to the increased endothelial permeability. Moreover, NGs and endothelial cells may also have a (pre)activated state that promotes the adhesion [40],

\section{Weaknesses of the Study}

The increased number of NGs and activation might have been underestimated. Our result refers only to the processes taking place in the circulation; thus, the confirmation of the pathogenic role of NGs in the tissues during HAE attacks requires further investigation. We had limited (8 and 14 samples) from patients during EM and HAE attack, respectively. Despite of the unique approach, we have just one patient with one attack in the attack kinetics follow-up part.

This study was focusing on NGs as supporting cells in the pathomechanism of C1-INH-HAE. The finding has brought to light new findings but raises questions as well. Apparently, C1-INH-HAE pathology is more complex than thought before. It raises a "chicken or egg" causality dilemma: are the NGs activated first and causing local edema, or the edema processes activates NGs? 
Table 4 Between-group difference of the measured cell counts and levels of factors related to neutrophil activation

\begin{tabular}{|c|c|c|c|c|c|}
\hline & $\begin{array}{c}\text { Symptom-free } \\
\text { patients } \\
\text { compared to } \\
\text { healthy } \\
\text { controls } \\
(n=77 / 79) \\
\end{array}$ & $\begin{array}{c}\text { EM } \\
\text { compared } \\
\text { to } \\
\text { symptom- } \\
\text { free period } \\
(n=8) \\
\end{array}$ & $\begin{array}{c}\text { HAE attack } \\
\text { compared } \\
\text { to } \\
\text { symptom- } \\
\text { free period } \\
(n=14) \\
\end{array}$ & $\begin{array}{l}\text { Follow- } \\
\text { up part } \\
\text { of the } \\
\text { study } \\
(n=1)^{*}\end{array}$ & $\begin{array}{c}\text { Effects on } \\
\text { NGs }\end{array}$ \\
\hline WBC & $\uparrow$ & $\uparrow$ & $\uparrow$ & $\uparrow$ & \\
\hline Lymphocytes & $\uparrow$ & & $\uparrow$ & & \\
\hline \multicolumn{6}{|l|}{ Thrombocytes } \\
\hline Monocytes & & $\uparrow$ & & $\uparrow$ & \\
\hline $\begin{array}{l}\text { Neutrophil } \\
\text { granulocytes }\end{array}$ & $\uparrow$ & & $\uparrow$ & $\uparrow$ & \\
\hline \multicolumn{6}{|l|}{$\begin{array}{l}\text { Eosinophil } \\
\text { granulocytes }\end{array}$} \\
\hline $\begin{array}{l}\text { Basophil } \\
\text { granulocytes }\end{array}$ & $\uparrow$ & & & & \\
\hline CCL5 & $\uparrow$ & & & & \multirow{2}{*}{$\begin{array}{c}\text { (probably) } \\
\text { no effects } \\
\text { on NGs }\end{array}$} \\
\hline MCP-1 & $\uparrow$ & & $\uparrow$ & & \\
\hline \multicolumn{5}{|l|}{ TNF a } & \multirow{3}{*}{$\begin{array}{c}\text { chemotactic } \\
\text { factors for } \\
\text { NGs }\end{array}$} \\
\hline IL-8 & & $\uparrow$ & & $\uparrow$ & \\
\hline LTB4 & & & $\uparrow$ & & \\
\hline NE & & & $\uparrow$ & & \multirow{4}{*}{$\begin{array}{c}\text { produced } \\
\text { by } \\
\text { activated } \\
\text { NGs }\end{array}$} \\
\hline NET & & & & $\uparrow$ & \\
\hline MPO & $\uparrow$ & & $\uparrow$ & & \\
\hline PRTN3 & & & & $\bar{\uparrow}$ & \\
\hline
\end{tabular}

$\uparrow$ means significantly higher/elevated cell count/level.

Symptom-free periods are marked in green, prodromal phase/EM in yellow, and HAE attack phase with orange.

*Note that this dataset from a single patient does not allow statistical assessment, suggesting only a trend. 


\section{Conclusion}

The alterations of NGC found in C1-INH-HAE patients may be driven by several factors, depending on the genotype, environmental conditions, nature of the triggering factor, and the actual molecular processes of the pathogenesis. These observations strongly support the hypothesis that $\mathrm{C} 1-\mathrm{INH}-$ HAE has a multifactorial pathomechanisms, beyond the monogenetic mutation in SERPING1 gene.

Author contributions EK-laboratory assays, sampling, measurement of neutrophil activation parameters, statistical analyses writing of the manuscript; NV—sampling, measurement of neutrophil activation parameters; BM and ZJ - measurement of neutrophil activation parameters; KVK - medical supervision of blood sampling; ALF-laboratory assays; LC - laboratory assays, statistical analyses, participated in writing of the manuscript; $\mathrm{LV}$ - provided data on patients and participated in the blood sampling; HF- head of the center, treating HAE patients, provided clinical data, designed and supervised the study, participated in writing of the manuscript; all authors contributed to the drafting or revision of the manuscript and provided their final approval of the manuscript for submission.

Funding Open access funding provided by Semmelweis University. This study was supported by the National Research, Development and Innovation Office, Hungary (K124557).

\section{Declarations}

Ethics Approval The study protocol was approved by the institutional review board of Semmelweis University of Budapest, and informed consent was obtained from all the participants in accordance with the Declaration of Helsinki.

Conflict of Interest KVK received honoraria and travel grants from CSL Behring and Shire and has participated in clinical trials of BioCryst, CSL Behring, Pharming, and Shire. LV received travel grants from CSL Behring and Shire Human Genetic Therapies Inc. HF received honoraria and travel grants from CSL Behring, Shire/Takeda, Swedish Orphan Biovitrum, Octapharma, Kalvista and Pharming; and/ or served as a consultant for these companies and has participated in clinical trials/registries for BioCryst, CSL Behring, Pharming, and Shire. Other authors have declared no conflict of interest.

Open Access This article is licensed under a Creative Commons Attribution 4.0 International License, which permits use, sharing, adaptation, distribution and reproduction in any medium or format, as long as you give appropriate credit to the original author(s) and the source, provide a link to the Creative Commons licence, and indicate if changes were made. The images or other third party material in this article are included in the article's Creative Commons licence, unless indicated otherwise in a credit line to the material. If material is not included in the article's Creative Commons licence and your intended use is not permitted by statutory regulation or exceeds the permitted use, you will need to obtain permission directly from the copyright holder. To view a copy of this licence, visit http://creativecommons.org/licenses/by/4.0/.

\section{References}

1. Busse PJ, Christiansen SC (2020) Hereditary angioedema. The New England journal of medicine 382(12):1136-1148. https:// doi.org/10.1056/NEJMra1808012

2. Magerl M, Doumoulakis G, Kalkounou I, Weller K, Church MK, Kreuz W, Maurer M (2014) Characterization of prodromal symptoms in a large population of patients with hereditary angio-oedema. Clin Exp Dermatol 39(3):298-303. https://doi. org/10.1111/ced.12285

3. Kohalmi KV, Veszeli N, Cervenak L, Varga L, Farkas H (2017) A novel prophylaxis with $\mathrm{C} 1$-inhibitor concentrate in hereditary angioedema during erythema marginatum. Immunol Lett 189:9093. https://doi.org/10.1016/j.imlet.2017.05.015

4. Rasmussen ER, de Freitas PV, Bygum A (2016) Urticaria and prodromal symptoms including erythema marginatum in Danish patients with hereditary angioedema. Acta Derm Venereol 96(3):373-376. https://doi.org/10.2340/00015555-2233

5. Germenis AE, Margaglione M, Pesquero JB, Farkas H, Cichon S, Csuka D, Lera AL, Rijavec M, Jolles S, Szilagyi A, Trascasa ML, Veronez CL, Drouet C, Zamanakou M, Hereditary Angioedema International Working G (2020) International consensus on the use of genetics in the management of hereditary angioedema. J Allergy Clin Immunol Pract 8(3):901-911. https://doi. org/10.1016/j.jaip.2019.10.004

6. Cugno M, Nussberger J, Cicardi M, Agostoni A (2003) Bradykinin and the pathophysiology of angioedema. Int Immunopharmacol 3(3):311-317. https://doi.org/10.1016/S1567-5769(02)00162-5

7. Kaplan AP (2010) Enzymatic pathways in the pathogenesis of hereditary angioedema: The role of $\mathrm{C} 1$ inhibitor therapy. $\mathrm{J}$ Allergy Clin Immunol 126 (5):918-925. https://doi.org/10.1016/j. jaci.2010.08.012

8. De Maat S, Hofman ZLM, Maas C (2018) Hereditary angioedema: the plasma contact system out of control. J Thromb Haemost : JTH 16(9):1674-1685. https://doi.org/10.1111/jth.14209

9. Wu MA, Bova M, Berra S, Senter R, Parolin D, Caccia S, Cicardi $M$ (2020) The central role of endothelium in hereditary angioedema due to $\mathrm{C} 1$ inhibitor deficiency. Int Immunopharmacol 82:106304. https://doi.org/10.1016/j.intimp.2020.106304

10. Koruth JS, Eckardt AJ, Levey JM (2005) Hereditary angioedema involving the colon: Endoscopic appearance and review of GI manifestations. Gastrointest Endosc 61(7):907-911

11. Kodama J, Uchida K, Yoshimura S, Katayama Y, Kushiro H, Yutani C, Funahashi S, Takamiya O, Matsumoto Y, Ando Y et al (1984) Studies of four Japanese families with hereditary angioneurotic edema: Simultaneous activation of plasma protease systems and exogenous triggering stimuli. Blut 49(5):405418. https://doi.org/10.1007/bf00319889

12. Goti F, Melcher GA, Spath P, Wuthrich B (1998) [Hereditary angioedema. A rare cause of acute abdominal pain with ascites]. Dtsch Med Wochenschr 123(40):1166-1171. https:// doi.org/10.1055/s-2007-1024139

13. Zotter Z, Csuka D, Varga L, Füst G, Farkas H (2010) WBC elevation and the resulting neutrophilia characterize hereditary angioedema attacks. Angioedema 1 (3).

14. Veszeli N, Csuka D, Zotter Z, Imreh E, Jozsi M, Benedek S, Varga L, Farkas H (2015) Neutrophil activation during attacks in patients with hereditary angioedema due to C1-inhibitor deficiency. Orphanet J Rare Dis 10:156. https://doi.org/10.1186/ s13023-015-0374-y

15. Henderson LM, Figueroa CD, Muller-Esterl W, Bhoola KD (1994) Assembly of contact-phase factors on the surface of the human neutrophil membrane. Blood 84(2):474-482 
16. Doring G (1994) The role of neutrophil elastase in chronic inflammation. Am J Respir Crit Care Med 150(6 Pt 2):S114117. https://doi.org/10.1164/ajrccm/150.6_Pt_2.S114

17. Belaaouaj A, Kim KS, Shapiro SD (2000) Degradation of outer membrane protein A in Escherichia coli killing by neutrophil elastase. Science 289(5482):1185-1188. https://doi. org/10.1126/science.289.5482.1185

18. de Agostini A, Patston PA, Marottoli V, Carrel S, Harpel PC, Schapira M (1988) A common neoepitope is created when the reactive center of $\mathrm{C} 1$-inhibitor is cleaved by plasma kallikrein, activated factor XII fragment, $\mathrm{C} 1$ esterase, or neutrophil elastase. J Clin Investig 82(2):700-705. https://doi.org/10.1172/JCI113650

19. Kinkade JM Jr, Pember SO, Barnes KC, Shapira R, Spitznagel JK, Martin LE (1983) Differential distribution of distinct forms of myeloperoxidase in different azurophilic granule subpopulations from human neutrophils. Biochem Biophys Res Commun 114(1):296-303. https://doi.org/10.1016/0006-291x(83)91627-3

20. Aratani Y (2018) Myeloperoxidase: Its role for host defense, inflammation, and neutrophil function. Arch Biochem Biophys 640:47-52. https://doi.org/10.1016/j.abb.2018.01.004

21. Radice A, Sabadini E, Sinico RA (2007) Antineutrophil Cytoplasmic Autoantibodies with Specificity for Proteinase 3. Autoantibodies, 2nd edn. pp 105-110. https://doi.org/10.1016/B978044452763-9/50018-4

22. Delgado-Rizo V, Martinez-Guzman MA, Iniguez-Gutierrez L, Garcia-Orozco A, Alvarado-Navarro A, Fafutis-Morris M (2017) Neutrophil extracellular traps and its implications in inflammation: An overview. Front Immunol 8:81. https://doi.org/10.3389/ fimmu.2017.00081

23. Jorch SK, Kubes P (2017) An emerging role for neutrophil extracellular traps in noninfectious disease. Nat Med 23(3):279-287. https://doi.org/10.1038/nm.4294

24. Kaplan MJ, Radic M (2012) Neutrophil extracellular traps: Double-edged swords of innate immunity. J Immunol 189(6):26892695. https://doi.org/10.4049/jimmunol.1201719

25. Leffler J, Martin M, Gullstrand B, Tyden H, Lood C, Truedsson L, Bengtsson AA, Blom AM (2012) Neutrophil extracellular traps that are not degraded in systemic lupus erythematosus activate complement exacerbating the disease. J Immunol 188(7):35223531. https://doi.org/10.4049/jimmunol.1102404

26. Oehmcke S, Morgelin M, Herwald H (2009) Activation of the human contact system on neutrophil extracellular traps. J Innate Immun 1(3):225-230. https://doi.org/10.1159/000203700

27. Ravussin E, Smith SR (2016) Role of the Adipocyte in Metabolism and Endocrine Function, vol Volume I. Endocrinology: Adult and Pediatric (Seventh Edition). Science Direct. https:// doi.org/10.1016/B978-0-323-18907-1.00036-6

28. Wong HR, Nowak JE, Standage SW, Oliveira CFd (2011) Sepsis. In: Fuhrman BP, Zimmerman JJ (eds) Pediatric Critical Care. 4th Edition edn. Science Direct. https://doi.org/10.1016/B978-0-32307307-3.10103-X

29. Feng P, Jyotaki M, Kim A, Chai J, Simon N, Zhou M, Bachmanov AA, Huang L, Wang H (2015) Regulation of bitter taste responses by tumor necrosis factor. Brain Behav Immun 49:32-42. https:// doi.org/10.1016/j.bbi.2015.04.001

30. Fitzgerald KA, O'Neill LAJ, Gearing AJH, Callard RE (2001) IL-8. The Cytokine FactsBook and Webfacts (Second Edition).

31. Louis NA, Parkos CA (2015) The Neutrophil, vol. Volume 1. Mucosal Immunol (Fourth Edition).

32. Veszeli N, Kohalmi KV, Kajdacsi E, Gulyas D, Temesszentandrasi G, Cervenak L, Farkas H, Varga L (2018) Complete kinetic follow-up of symptoms and complement parameters during a hereditary angioedema attack. Allergy 73(2):516-520. https:// doi.org/10.1111/all.13327

33. Song DY, Gu JY, Yoo HJ, Kim YI, Nam-Goong IS, Kim ES, Kim HK (2019) Activation of factor XII and kallikrein-kinin system combined with neutrophil extracellular trap formation in diabetic retinopathy. Exp Clin Endocrinol Diabetes. https://doi. org/10.1055/a-0981-6023

34. Khanfer R, Carroll D, Lord JM, Phillips AC (2012) Reduced neutrophil superoxide production among healthy older adults in response to acute psychological stress. Int J Psychol : Official Journal of the International Organization of Psychophysiology 86(3):238-244. https://doi.org/10.1016/j.ijpsycho.2012.09.013

35. Khanfer R, Phillips AC, Carroll D, Lord JM (2010) Altered human neutrophil function in response to acute psychological stress. Psychosom Med 72(7):636-640. https://doi.org/10.1097/PSY.0b013 e3181e7fae8

36. Zotter Z, Csuka D, Szabo E, Czaller I, Nebenfuhrer Z, Temesszentandrasi G, Fust G, Varga L, Farkas H (2014) The influence of trigger factors on hereditary angioedema due to C1-inhibitor deficiency. Orphanet J Rare Dis 9(1):44. https://doi. org/10.1186/1750-1172-9-44

37. Hallsworth MP, Soh CP, Lane SJ, Arm JP, Lee TH (1994) Selective enhancement of GM-CSF, TNF-alpha, IL-1 beta and IL-8 production by monocytes and macrophages of asthmatic subjects. Eur Respir J 7(6):1096-1102

38. Lukacs NW, Strieter RM, Elner V, Evanoff HL, Burdick MD, Kunkel SL (1995) Production of chemokines, interleukin-8 and monocyte chemoattractant protein-1, during monocyte: Endothelial cell interactions. Blood 86(7):2767-2773

39. Harada A, Sekido N, Akahoshi T, Wada T, Mukaida N, Matsushima K (1994) Essential involvement of interleukin-8 (IL-8) in acute inflammation. J Leukoc Biol 56(5):559-564

40. Kajdacsi E, Jani PK, Csuka D, Varga LA, Prohaszka Z, Farkas H, Cervenak L (2014) Endothelial cell activation during edematous attacks of hereditary angioedema types I and II. J Allergy Clin Immunol 133(6):1686-1691. https://doi.org/10.1016/j. jaci.2013.12.1072

41. Ehrenfeld P, Bhoola KD, Matus CE, Figueroa CD (2018) Functional interrelationships between the kallikrein-related peptidases family and the classical kinin system in the human neutrophil. Biol Chem 399(9):925-935. https://doi.org/10.1515/hsz-2017-0338

42. Kajdacsi E, Jandrasics Z, Veszeli N, Mako V, Koncz A, Gulyas D, Kohalmi KV, Temesszentandrasi G, Cervenak L, Gal P, Dobo J, de Maat S, Maas C, Farkas H, Varga L (2020) Patterns of C1-inhibitor/plasma serine protease complexes in healthy humans and in hereditary angioedema patients. Front Immunol 11:794. https://doi.org/10.3389/fimmu.2020.00794

43. Balamayooran G, Batra S, Balamayooran T, Cai S, Jeyaseelan S (2011) Monocyte chemoattractant protein 1 regulates pulmonary host defense via neutrophil recruitment during Escherichia coli infection. Infect Immun 79(7):2567-2577. https://doi.org/10.1128/ IAI.00067-11

44. Di Stefano A, Caramori G, Gnemmi I, Contoli M, Bristot L, Capelli A, Ricciardolo FL, Magno F, D’Anna SE, Zanini A, Carbone M, Sabatini F, Usai C, Brun P, Chung KF, Barnes PJ, Papi A, Adcock IM, Balbi B (2009) Association of increased CCL5 and CXCL7 chemokine expression with neutrophil activation in severe stable COPD. Thorax 64(11):968-975. https://doi.org/10.1136/ thx.2009.113647

45. Grymova T, Vlkova M, Soucek P, Hakl R, Nechvatalova J, Slanina P, Stichova J, Litzman J, Freiberger T (2019) Neutrophils are dysregulated in patients with hereditary angioedema types I and II in a symptom-free period. Mediators Inflamm 2019:9515628. https:// doi.org/10.1155/2019/9515628

46. Kohalmi KV, Mezo B, Veszeli N, Benedek S, Feher A, Holdonner A, Jesenak M, Varga L, Farkas H (2020) Changes of coagulation parameters during erythema marginatum in patients with hereditary angioedema. Int Immunopharmacol 81:106293. https://doi. org/10.1016/j.intimp.2020.106293 
47. Nguyen A, Zuraw BL, Christiansen SC (2020) Contact system activation during erythema marginatum in hereditary angioedema. Ann Allergy Asthma Immunol : Official Publication of the American College of Allergy, Asthma, \& Immunology 124 (4):394-395 e391. https://doi.org/10.1016/j.anai.2020.01.009
Publisher's Note Springer Nature remains neutral with regard to jurisdictional claims in published maps and institutional affiliations. 